\title{
APPENDIX 3
}

\section{Shading Coefficients}

The shading coefficient formula is derived from McPherson et al. (2018). The shading coefficient (y) is based species-specific coefficients (Table 11) and d.b.h., based on the formula:

$$
y=0.0617^{\star} \ln (x)+0.615+\text { species-specific shading coefficient }
$$

Where: $\mathrm{x}=$ d.b.h in $\mathrm{cm}$.

Table 11.-Species-specific shading coefficients

\begin{tabular}{|c|c|c|c|}
\hline Species & Coefficient & Species & Coefficient \\
\hline Acacia farnesiana & 0.006476 & Cocos nucifera & -0.212762 \\
\hline Acacia melanoxylon & 0.033203 & Conocarpus erectus & -0.064823 \\
\hline Acacia salicina & 0.036297 & Cordia subcordata & -0.034285 \\
\hline Acer macrophyllum & 0.007330 & Cornus florida & -0.041082 \\
\hline Acernegundo & 0.013600 & Crataegus spp. & 0.005855 \\
\hline Acer palmatum & 0.019860 & Crataegus x lavallei & -0.044633 \\
\hline Acer platanoides & 0.038195 & Cupaniopsis anacardioides & -0.032094 \\
\hline Acer rubrum & 0.009591 & Delonix regia & -0.108310 \\
\hline Acer saccharinum & 0.017403 & Elaeagnus angustifolia & -0.000679 \\
\hline Acer saccharum & 0.024004 & Elaeodendron orientale & -0.021490 \\
\hline Bauhinia blakeana & -0.148861 & Eriobotrya japonica & -0.031987 \\
\hline Betula nigra & 0.018711 & Eucalyptus ficifolia & 0.008154 \\
\hline Betula pendula & 0.001379 & Eucalyptus globulus & -0.013452 \\
\hline Brachychiton populneus & 0.001286 & Eucalyptus microtheca & -0.005495 \\
\hline Callistemon citrinus & -0.000129 & Eucalyptus sideroxylon & 0.004389 \\
\hline Calocedrus decurrens & 0.030777 & Fagus sylvatica & 0.007078 \\
\hline Calophyllum inophyllum & -0.066124 & Ficus benjamina & -0.005748 \\
\hline Carpinus betulus & 0.064152 & Ficus thonningii & 0.019105 \\
\hline Carya illinoinensis & -0.015547 & Filicium decipiens & 0.036698 \\
\hline Cassia $\times$ nealiae & -0.052799 & Fraxinus americana & 0.040826 \\
\hline Casuarina equisetifolia & -0.187445 & Fraxinus angustifolia & 0.055648 \\
\hline Catalpa speciosa & 0.008864 & Fraxinus excelsior & 0.039979 \\
\hline Cedrus deodara & -0.021595 & Fraxinus holotricha & 0.021817 \\
\hline Celtis laevigata & -0.007073 & Fraxinus latifolia & 0.017034 \\
\hline Celtis occidentalis & 0.018599 & Fraxinus pennsylvanica & 0.031117 \\
\hline Celtis sinensis & 0.025890 & Fraxinus uhdei & -0.006909 \\
\hline Ceratonia siliqua & -0.020249 & Fraxinus velutina & 0.001310 \\
\hline Chilopsis linearis & 0.006679 & Ginkgo biloba & -0.014228 \\
\hline Cinnamomum camphora & 0.017635 & Gleditsia triacanthos & -0.009429 \\
\hline Citharexylum spinosum & -0.042121 & Gymnocladus dioicus & 0.048339 \\
\hline
\end{tabular}

Appendix 3 (https://doi.org/10.2737/NRS-GTR-200-2021-Appendix3) in: Nowak, David J. 2021. Understanding i-Tree: Summary of programs and methods. Gen. Tech. Rep. NRS-200-2021. Madison, WI: U.S. Department of Agriculture, Forest Service, Northern Research Station. 100 p. [plus 14 appendixes]. https://doi.org/10.2737/NRS-GTR-200-2021. 
Table 11.-Continued

\begin{tabular}{|c|c|}
\hline Species & Coefficient \\
\hline Ilex opaca & 0.030095 \\
\hline Ilex paraguayensis & -0.114463 \\
\hline Jacaranda mimosifolia & 0.005707 \\
\hline Juglans nigra & 0.003086 \\
\hline Juniperus virginiana & 0.030798 \\
\hline Koelreuteria elegans & -0.016586 \\
\hline Koelreuteria paniculata & 0.027350 \\
\hline Lagerstroemia indica & -0.014415 \\
\hline Lagerstroemia sp. & -0.076014 \\
\hline Liquidambar styraciflua & 0.021253 \\
\hline Liriodendron tulipifera & 0.027576 \\
\hline Magnolia grandiflora & 0.006197 \\
\hline Malus sp. & -0.013821 \\
\hline Melaleuca quinquenervia & -0.045497 \\
\hline Metrosideros excelsa & 0.035613 \\
\hline Morus alba & 0.001130 \\
\hline Olea europaea & 0.012154 \\
\hline Parkinsonia aculeata & -0.061333 \\
\hline Parkinsonia florida & -0.025384 \\
\hline Phoenix canariensis & -0.081700 \\
\hline Phoenix dactylifera & -0.175857 \\
\hline Picea pungens & 0.023440 \\
\hline Pinus brutia & 0.008550 \\
\hline Pinus canariensis & 0.012044 \\
\hline Pinus contorta & 0.012737 \\
\hline Pinus echinata & -0.039363 \\
\hline Pinus edulis & 0.032428 \\
\hline Pinus elliottii & -0.059779 \\
\hline Pinus halepensis & 0.017895 \\
\hline Pinus nigra & 0.033034 \\
\hline Pinus ponderosa & 0.040393 \\
\hline Pinus radiata & 0.002821 \\
\hline Pinus sylvestris & 0.014577 \\
\hline Pinus taeda & -0.015568 \\
\hline Pinus thunbergii & -0.010467 \\
\hline Pistacia chinensis & 0.030910 \\
\hline Pittosporum undulatum & -0.004301 \\
\hline Platanus occidentalis & 0.001356 \\
\hline Platanus racemosa & 0.029835 \\
\hline Platanus $x$ acerifolia & 0.013500 \\
\hline Platycladus orientalis & 0.036982 \\
\hline Podocarpus macrophyllus & -0.036235 \\
\hline Populus angustifolia & 0.018432 \\
\hline Populus fremontii & 0.011781 \\
\hline
\end{tabular}

\begin{tabular}{|c|c|}
\hline Species & Coefficient \\
\hline Prosopis chilensis & -0.050350 \\
\hline Prunus caroliniana & -0.000254 \\
\hline Prunus cerasifera & -0.002760 \\
\hline Prunus serrulata & -0.075394 \\
\hline Prunus sp. & 0.011707 \\
\hline Prunus yedoensis & -0.023468 \\
\hline Pseudotsuga menziesii & -0.017942 \\
\hline Pyrus calleryana & 0.028712 \\
\hline Pyrus kawakamii & -0.056219 \\
\hline Quercus alba & 0.018694 \\
\hline Quercus laurifolia & -0.003916 \\
\hline Quercus lobata & 0.000927 \\
\hline Quercus macrocarpa & 0.028163 \\
\hline Quercus nigra & -0.006829 \\
\hline Quercus palustris & -0.011858 \\
\hline Quercus phellos & 0.005547 \\
\hline Quercus rubra & 0.022225 \\
\hline Quercus shumardii & 0.009522 \\
\hline Quercus agrifolia & 0.021636 \\
\hline Quercusilex & 0.024552 \\
\hline Quercus virginiana & -0.016080 \\
\hline Rhus lancea & -0.031603 \\
\hline Robinia pseudoacacia & -0.023624 \\
\hline Samanea saman & -0.048322 \\
\hline Schinus molle & 0.027216 \\
\hline Schinus terebinthifolia & 0.019735 \\
\hline Sequoia sempervirens & 0.003386 \\
\hline Swietenia mahogani & -0.032240 \\
\hline Tabebuia aurea & -0.048674 \\
\hline Tabebuia heterophylla & -0.061165 \\
\hline $\begin{array}{l}\text { Tabebuia ochracea ssp. } \\
\text { neochrysantha }\end{array}$ & -0.081893 \\
\hline Tilia americana & 0.043399 \\
\hline Tilia cordata & 0.044529 \\
\hline Triadica sebifera & -0.031487 \\
\hline Tristaniopsis conferta & -0.036760 \\
\hline Ulmus alata & -0.010829 \\
\hline Ulmus americana & -0.016822 \\
\hline Ulmus parvifolia & -0.024810 \\
\hline Ulmus pumila & 0.009616 \\
\hline Veitchia merrillii & -0.105305 \\
\hline Washingtonia filifera & -0.144123 \\
\hline Washingtonia robusta & -0.085184 \\
\hline Zelkova serrata & 0.016636 \\
\hline
\end{tabular}

